ISSN: $2675-9683$

\title{
Deficiência de Antitrombina na Gestação - Uma Revisão de Literatura
}

\section{Antithrombin Deficiency in Gestation - A Literature Review}

\author{
Jandir Mendonça Nicacio ${ }^{1}$, Leonardo Fernandes e Santana ${ }^{1}$, Mateus de Sousa Rodrigues ${ }^{1}$, \\ Manoel Pereira Guimarães ${ }^{1}$, Orlando Vieira Gomes ${ }^{1}$, Stênio Galvão de Freitas ${ }^{2,3}$, Sindara \\ Nunes Parente ${ }^{2,3}$, Marcelo Marques de Souza Lima ${ }^{2,3}$ \\ ${ }^{1}$ Universidade Federal do Vale do São Francisco (UNIVASF) - Petrolina (PE), Brasil ${ }^{2}$ Hospital Dom Malan - \\ Petrolina (PE), Brasil ${ }^{3}$ Instituto de Medicina Integral Professor Fernando Figueira (IMIP) - Petrolina (PE), \\ Brasil
}

E-mail para contato: manoelpeguimaraes@gmail.com

Artigo recebido em 01/12/2020 e aceito em 10/02/2021

\begin{abstract}
RESUMO
A antitrombina é um anticoagulante endógeno responsável por inibir uma série de enzimas envolvidas na cascata de coagulação. Sua deficiência é rara, foi descrita pela primeira vez em 1965, tem caráter autossômico dominante, e está associada à trombose familiar. Este estudo trata-se de uma revisão narrativa de literatura realizada nas bases de dados do PubMed Central (PMC), da MEDLINE e da COCHRANE. Ao todo, 12 artigos foram elegíveis em nossa pesquisa. A análise das publicações indica que a antitrombina é um anticoagulante endógeno que inativa uma série de fatores de coagulação. Sua deficiência é rara, mas aumenta significativamente o risco de eventos tromboembólicos, principalmente na gestação. O diagnóstico dessa desordem e o conhecimento da efetividade do tratamento são os primeiros passos para reduzir os eventos adversos desta doença na gestação.
\end{abstract}

Palavras-chave: Gestação; Deficiência de Antitrombina III; Trombofilia.

\section{ABSTRACT}

Antithrombin is an endogenous anticoagulant responsible for inhibiting a series of enzymes involved in the coagulation cascade. Its deficiency is rare, it was first described in 1965, has an autosomal dominant character, and is associated with familial thrombosis. This study is a narrative literature review carried in PubMed Central (PMC), MEDLINE and COCHRANE databases. In all of them, 12 articles were eligible for our research. Analysis of these studies indicates that antithrombin is an endogenous anticoagulant that inactivates a number of coagulation factors. Its deficiency is rare, but it significantly increases the risk of thromboembolic events, especially during pregnancy. The diagnosis of this disorder and the knowledge of the effectiveness of the treatment are the first steps to reduce the adverse events of this disease during pregnancy.

Keywords: Pregnancy; Antithrombin III Deficiency; Thrombophilia.

\section{INTRODUÇÃO}

A antitrombina (AT) é um anticoagulante endógeno que inibe muitas enzimas da cascata de coagulação. Sua deficiência hereditária é rara, tem caráter autossômico dominante, e foi descrita pela primeira vez em 1965 por Egeberg, ${ }^{1}$ que demonstrou uma associação entre baixos níveis plasmáticos de AT e trombose familiar. ${ }^{2-7} \mathrm{O}$ primeiro defeito funcional identificado da AT recebeu o nome de AT Budapest. ${ }^{3}$

A prevalência estimada da deficiência de AT varia de 1:500 a 1:5000 na população geral, e aumenta para escalas de 1:20 a 1:200 em famílias com histórico de tromboembolismo venoso 
(TEV). ${ }^{3,5,8-10}$ Em gestantes, há uma estimativa de risco anteparto e pós-parto de 3\% a 17,7\% nos estudos mais recentes. ${ }^{2,8,11,12}$ Acredita-se que sua incidência seja menor que a da deficiência de proteína $\mathrm{C}$, de proteína $\mathrm{S}$, da mutação do fator $\mathrm{V}$ de Leiden, e da mutação G20210A da protrombina. ${ }^{2}$ De todas as desordens trombofílicas hereditárias, a deficiência de AT é a que confere maior risco de TEV. ${ }^{2,5,12}$

A AT é um inibidor de serino-protease (SERPIN) sintetizado no fígado que inativa múltiplas enzimas geradas pela cascata de coagulação. Seus principais alvos são os fatores IIa (trombina), Xa, e IXa e, em menor extensão, os fatores XIa e XIIa, bem como a calicreína e a plasmina. ${ }^{2,5,8,12}$ Apresenta papel na inibição do fator tecidual e na inativação do complexo VIIafator tecidual, além de prevenir indiretamente a ativação da proteína $\mathrm{C}$ ao inibir a trombina. A proteína $\mathrm{C}$ ativada forma um complexo com a proteína $\mathrm{S}$ livre na presença de cálcio na superfície da plaqueta ativada para inibir os fatores VIIIa e Va. A proteólise dos fatores VIIIa e $\mathrm{Va}$ previne a ativação do fator $\mathrm{X}$ e da protrombina, respectivamente, o que limita a produção de trombina. ${ }^{2,5,10,12}$

Mais de 250 mutações no gene da AT foram descritas. O gene da AT (SERPINC1) está localizado no cromossomo 1q23-25.,2,5,7,12 A maioria dos defeitos genéticos resulta em risco trombótico elevado. A homozigose na deficiência de AT tipo I, e na maioria dos casos de deficiência do tipo II, com exceção das variantes que afetam o sítio de ligação da heparina (heparin binding site, HBS), são incompatíveis com a vida. ${ }^{5}$

A deficiência de AT tipo I (quantitativa) afeta quantitativamente os níveis da atividade da AT. É mais comumente causado por mutações missense (de sentido trocado), deleções frameshift, mutações nonsense (sem sentido), pequenas inserções ou deleções (de menos de 30 pares de bases), além de outras deleções maiores. O tipo II (qualitativo) é dividido em 3 subtipos (IIa, IIb e IIc). É caracterizado por mutações que afetam o sítio reativo da AT (tipo IIa), do domínio de ligação da heparina (tipo IIb), e inclui um grupo pleomórfico de mutações próximo ao laço reativo que resulta em decréscimo da atividade da AT (tipo IIc). 2,5,11

Destarte, essa revisão narrativa da literatura tem o objetivo de atualizar os dados disponíveis sobre a deficiência de AT na gestação publicados em um período de cinco anos. Para isso, o presente trabalho é composto de uma revisão da literatura dos textos científicos publicados entre 2014 e 2018 acerca da deficiência de antitrombina em gestantes. A proposta da revisão é compactar os estudos sobre o tema, de modo a analisar o comportamento de tal entidade nosológica no contexto da gestação, encerrando uma compreensão ampla sobre a doença. A pesquisa foi realizada em outubro de 2018, e contempla as bases de dados do PubMed Central (PMC), da MEDLINE e da COCHRANE. Foram utilizados os Mesh Terms "Antithrombin III deficiency" e "Pregnancy", utilizando o operador Booleano "AND". No PubMed, foram encontrados 186 resultados, os quais foram reduzidos a 16 itens com a aplicação do filtro de temporalidade. Foram incluídos todos os artigos encontrados. Foram excluídos os textos duplicados e os que se tratavam de relato de caso. $\mathrm{Na}$ COCHRANE, nenhuma meta-análise ou revisão sistemática foi publicada entre 2014 e 2018.

\section{DESENVOLVIMENTO}

\section{Gestação e risco de Trombose na Deficiência de Antitrombina}

A TEV está primariamente associada à deficiência de AT, principalmente de membros inferiores e pulmonar. Já a trombose arterial raramente ocorre em pacientes com deficiência de AT, uma vez que a maioria dos eventos oclusivos nas artérias ocorre no contexto de arteriosclerose subjacente. Em uma coorte italiana, Martinelli e colaboradores verificaram um risco condicional de TEV de 42,8 em pacientes com deficiência de antitrombina $(\mathrm{CI}=95 \% ; 10,2-180,3) .{ }^{13}$ Também foi observado que a incidência de TEV em familiares de pacientes com deficiência de antitrombina é maior em comparação à incidência de TEV na população normal $(76,5 \%$ x $3,6 \%)$, com uma incidência anual de $2,82 \%$ e de $0,10 \%$ respectivamente. Outra coorte italiana realizada em pacientes com trombofilias hereditárias verificou que a incidência de TEV está associada à idade, mas não ao sexo, com idade média de acometimento entre 30 e 40 anos. Na gestação, o risco de TEV é maior no pós-parto (21\%) do que durante a gestação $(18 \%){ }^{2}$ O risco de TEV varia, de acordo com o tipo de deficiência de AT, sendo menor no tipo IIb.

Em 1856, Virchow descreveu os três pilares da trombose: lesão endotelial, estase sanguínea, e hipercoagulabilidade. Todos esses elementos estão presentes na gravidez. As alterações de fluxo sanguíneo na gravidez resultam em estase venosa, e a lesão endotelial pode ocorrer durante o trabalho de parto. ${ }^{6} \mathrm{~A}$ deficiência de AT na gestação aumenta o risco de complicações tromboembólicas em até seis vezes, 
além de aumentar o risco de perda fetal. ${ }^{11,12}$ Trombose complica aproximadamente $37 \%$ das gestações e dos partos em mulheres com deficiência de AT. Há risco aumentado para: préeclâmpsia, natimortalidade, restrição de crescimento intrauterino, ruptura de placenta, hemólise, elevação de enzimas hepáticas e plaquetopenia (síndrome HELLP)., ${ }^{4,6,9,12}$ Já foi descrito que a administração de AT durante o trabalho de parto foi eficaz em prevenir recorrências trombóticas no contexto da deficiência de AT, em pacientes com história de trombose recente ou recorrente. ${ }^{2} \mathrm{Na}$ Alemanha, mais de 1000 mulheres com TEV associada à gestação foram rastreadas para trombofilias hereditárias ou adquiridas em um estudo multicêntrico e retrospectivo. Um total de sete pacientes com deficiência de AT foi identificado, em um total de 18 gestações, dos quais foram observados: 11 neonatos saudáveis com $\geq 37$ semanas de gestação, um neonato prematuro com 25 semanas de gestação, duas perdas fetais com 21 e 28 semanas de gestação, e quatro abortos espontâneos. ${ }^{2}$ Foram observados três eventos tromboembólicos nessas pacientes durante a gestação, e, apesar da instituição de terapia com heparina de baixo peso molecular (HBPM), um evento ocorreu no pós-parto. No entanto, o estudo observou menores taxas de complicações, bem como melhores desfechos neonatais quando a HBPM foi utilizada combinada ao concentrado de $\mathrm{AT}^{2}$

\section{Diagnóstico da Deficiência de Antitrombina}

A deficiência de AT é comumente suspeitada quando o paciente apresenta histórico familiar de TEV em indivíduos com menos de 50 anos, na ausência de fatores de risco como cirurgia, câncer e imobilização, e na presença de diminuição dos níveis plasmáticos de AT. Com o decréscimo da concentração de AT, o paciente pode se tornar resistente à heparina, mesmo a doses elevadas. Portanto, a resistência à heparina pode ser um sinal de deficiência de AT. ${ }^{2,8}$ Devem ser lembrados os diagnósticos diferenciais de causas adquiridas de deficiência de AT, como os apresentados no quadro 1 .

Quadro 1: Causas adquiridas de deficiência de antitrombina

\begin{tabular}{|c|c|}
\hline MECANISMO & EXEMPLOS \\
\hline Diminuição da síntese de AT & $\begin{array}{ll}- & \text { Hepatopatia } \\
- & \text { Desnutrição status neonatal }\end{array}$ \\
\hline Aumento da excreção de AT & $\begin{array}{ll}\text { - } & \text { Síndrome nefrótica } \\
\text { - } & \text { Síndrome do intestino irritável } \\
\text { - } & \text { Enteropatia perdedora de proteínas } \\
& \text { Diabetes mellitus. }\end{array}$ \\
\hline Consumo acelerado de AT & $\begin{array}{ll}- & \text { Coagulação intravascular disseminada } \\
\text { - } & \text { Cirurgia } \\
\text { - } & \text { Queimaduras } \\
\text { - } & \text { Sepse } \\
\text { - } & \text { Doença inflamatória intestinal } \\
\text { - } & \text { Trombose aguda, } \\
\text { - } & \text { Pré-eclâmpsia } \\
\end{array}$ \\
\hline $\begin{array}{l}\text { Depleção de AT induzida por } \\
\text { drogas }\end{array}$ & $\begin{array}{ll} & \text { Heparina } \\
- & \text { L-asparginase } \\
- & \text { Monometoxipolietilenoglicol asparginase } \\
\text { - } & \text { Contraceptivos orais }\end{array}$ \\
\hline Outros & $\begin{array}{ll}- & \text { Plasmaférese } \\
\text { - } & \text { Hemodiálise } \\
\text { - } & \text { Bypass cardiopulmonar } \\
\text { - } & \text { Membrana de oxigenação extracorpórea } \\
\text { - } & \text { Doença de Behçet } \\
\text { - } & \text { Pós-infusão de concentrado de protrombina } \\
\text { - } & \text { Malignidade } \\
\text { Idade avançada }\end{array}$ \\
\hline
\end{tabular}

Nota: AT: antitrombina 
Ensaios funcionais para diagnosticar a deficiência de AT incluem o ensaio de atividade cromogênica, no qual o plasma do paciente é incubado na presença de heparina com excesso de trombina ou de fator Xa. No entanto, esse tipo de ensaio funcional não diferencia o tipo IIa do tipo IIb de deficiência. A detecção do tipo IIb pode ser realizada a partir de um ensaio de atividade de AT executado na ausência de heparina ou a partir de sequenciamento genético. ${ }^{2} \mathrm{O}$ tipo de deficiência de AT também pode ser identificado através análise genética e de ensaios antigênicos (Quadro 1). 2,5

Manejo de pacientes com Deficiência de Antitrombina

A gravidez constitui um dos fatores de risco adicionais (ex. cirurgia, imobilização, câncer) que nos leva a considerar a profilaxia com concentrado de AT em pacientes com deficiência de AT e histórico de TEV. Durante o evento agudo de TEV e/ou resistência à heparina, a terapia de reposição de AT pode ser utilizada em associação à heparina no hospital. Em raras ocasiões, um paciente com deficiência de AT pode precisar de suplementação de AT ambulatorialmente enquanto também recebe HBPM. ${ }^{2,6}$

A AT humana derivada de plasma (pdAT; Thrombate III, Grifols) e a AT humana recombinante (rhAT; ATryn, rEVO Biologics) foram aprovadas pelo Food and Drug Administration (FDA). $\mathrm{O}$ concentrado de antitrombina está indicado para prevenir o TEV em pacientes com deficiência de AT. Nessas situações, o objetivo é aumentar a atividade da AT para o normal (80\%-120\%). ${ }^{2,10}$

Ainda não existem grandes ensaios clínicos randomizados e diretrizes para estabelecer com precisão a melhor conduta diante de gestantes com deficiência de AT. A recomendação é que a gestante seja acompanhada por especialistas e seja avaliada individualmente. As evidências mais atuais sugerem as seguintes condutas:

1) Tromboembolismo venoso agudo: concentrados de AT podem ser utilizados para normalizar os níveis de AT. ${ }^{8}$ 2) História de tromboembolismo em pacientes anticoagulados: essas gestantes devem receber doses plenas de HBPM durante a gestação e o período pós-parto. O concentrado de AT é uma opção quando a anticoagulação está associada a alto risco de sangramento, em casos de cirurgias ou procedimentos invasivos, de abortamento espontâneo, ou durante o parto. ${ }^{8}$
3) História de tromboembolismo em pacientes não anticoagulados: a confirmação diagnóstica é crítica. Em casos de atividade de AT $<60 \%$, a paciente deve ser tratada como se tivesse história de TEV em anticoagulação crônica: receber doses plenas de HBPM durante a gestação e o puerpério. $^{2,8}$ 4) Atividade de AT $<60 \%$ em pacientes sem história pessoal ou familiar de TEV: nessa situação podemos apenas observar a paciente ou fazer anticoagulação profilática dependendo de outros fatores de risco e das preferências da paciente. Concentrados de AT só são prescritos nessa situação se a atividade de AT estiver muito baixa $(<40 \%){ }^{2,8}$

O concentrado de AT humana é administrado via intravenosa em uma dose inicial calculada a partir da atividade de AT basal. A dose inicial é dada em 10 a 20 minutos, e a atividade de AT deve ser monitorada inicialmente após 12 horas da dose inicial e antes da próxima infusão. Doses de manutenção são ajustadas de acordo com os níveis de AT de cada paciente. ${ }^{10}$ A AT humana recombinante é indicada para prevenção, mas não para tratamento, de TEV perioperatória ou periparto em pacientes com deficiência de AT. Ao contrário da pdAT, a meia-vida da rhAT é bem menor e requer administração contínua via bomba de infusão. ${ }^{10}$

A decisão de tratar com pdAT ou rhAT deve ser avaliada individualmente em cada paciente, considerando a dose total e a duração do tratamento. Para gestantes com deficiência de AT que desenvolvem TEV, HBPM e/ou heparina não fracionada (HNF) administrada via subcutânea são opções durante a gestação. Para gestantes com perfil de alto risco para TEV (ex. antecedentes de TEV) com níveis de AT < $60 \%$ e aquelas com níveis de AT < 40\%, o concentrado de AT deve ser administrado imediatamente antes e durante (em caso de rhAT) o trabalho de parto, quando outros anticoagulantes não podem ser administrados. ${ }^{2,8,12}$

\section{Prognóstico}

Trata-se de uma trombofilia de alto risco que poderia levar à trombose da circulação uterina ou intervilosa, o que contribuiria para hipoperfusão fetal independente de $\mathrm{o}$ feto apresentar ou não deficiência de $\mathrm{AT}^{4}{ }^{4} \mathrm{Em}$ uma revisão sistemática que incluiu um estudo para cada evento adverso (abortamento precoce, abortamento tardio, natimortalidade e préeclâmpsia), não houve evidência de relação entre esses desfechos e a deficiência de AT. Entretanto, tal associação foi observada em séries de casos posteriores, principalmente nos casos que não 
receberam tromboprofilaxia. Em uma série de casos de 12 gestantes, não houve perda fetal no grupo que recebeu tromboprofilaxia, mas houve em $63 \%$ daquelas que não receberam. Em outra série que incluiu 18 gestações, foi observada diferença na taxa de restrição de crescimento fetal entre aquelas que receberam e que não receberam a tromboprofilaxia (27\% e $50 \%$ respectivamente), bem como diferença na taxa de natimortalidade (0\% e $50 \%$ respectivamente) ${ }^{8}$

Por se tratar de uma revisão narrativa, o presente estudo possuí limitações. Podem ocorrer vieses de seleção e a avaliação dos trabalhos pode ser realizada com critérios desconhecidos. Esse tipo de revisão não é rigorosamente reprodutível..$^{14}$ No entanto, o trabalho cumpre seu papel de revisar o assunto.

\section{CONCLUSÃO}

Antitrombina é um anticoagulante endógeno que inativa uma série de fatores de coagulação. Sua deficiência é rara, mas é uma condição que aumenta significativamente o risco de eventos tromboembólicos, complicando ainda mais o estado trombofílico gestacional. Devido às complicações materno-fetais associadas à deficiência de AT, especialmente sem o tratamento, o diagnóstico dessa desordem e o conhecimento da efetividade do tratamento são os primeiros passos para reduzir os eventos adversos desta doença na gestação.

\section{REFERÊNCIAS}

1. Egeberg O. Inherited antithrombin deficiency causing thrombophilia. Thromb Diath Haemorrh. 1965;13:516-30.

2. Bauer KA, Nguyen-Cao TM, Spears JB. Issues in the Diagnosis and Management of Hereditary Antithrombin Deficiency. Ann Pharmacother. 2016;50(9):758-67.

http://dx.doi.org/10.1177/1060028016651276

3. Gindele R, Oláh Z, Ilonczai $P$, Speker $M$, Udvari Á, Selmeczi A, et al. Founder effect is responsible for the p.Leu131Phe heparin-bindingsite antithrombin mutation common in Hungary: phenotype analysis in a large cohort. J Thromb Haemost. 2016;14(4):704-15. http://dx.doi.org/10.1111/jth.13252

4. Kraft J, Sunder-Plassmann R, Mannhalter C, Quehenberger P, Tews G, Langer M, et al. Women with homozygous AT deficiency type II heparinbinding site (HBS) are at high risk of pregnancy loss and pregnancy complications. Ann Hematol.
2017;96(6):1023-31. http://dx.doi.org/10.1007/s00277-017-2965-2

5. Marshall AL, Botero JP, Ashrani AA, Pruthi RK, Heit JA, Chintakuntlawar A, et al. The Impact of Antithrombin Deficiency on Women's Reproductive Health Experiences and Healthcare Decision-Making. J Womens Health (Larchmt). 2017;26(12):1350-5.

http://dx.doi.org/10.1089/jwh.2017.6333

6. Rogenhofer N, Bohlmann MK, Beuter-Winkler P, Würfel W, Rank A, Thaler CJ, et al. Prevention, management and extent of adverse pregnancy outcomes in women with hereditary antithrombin deficiency. Ann Hematol. 2014;93(3):385-92. http://dx.doi.org/10.1007/s00277-013-1892-0

7. Sharma A, Bhakuni T, Ranjan R, Kumar R, Kishor K, Kamal VK, et al. Polymorphisms in factor $\mathrm{V}$ and antithrombin III gene in recurrent pregnancy loss: a case-control study in Indian population. J Thromb Thrombolysis. 2015;39(4):481-8.

http://dx.doi.org/10.1007/s11239-015-1186-6

8. James AH, Bates SM, Bauer KA, Branch W, Mann K, Paidas M, et al. Management of hereditary antithrombin deficiency in pregnancy. Thromb Res. 2017;157:41-5. http://dx.doi.org/10.1016/j.thromres.2017.05.017 9. Paidas MJ, Forsyth C, Quéré I, Rodger M, Frieling JTM, Tait RC. Perioperative and peripartum prevention of venous thromboembolism in patients with hereditary antithrombin deficiency using recombinant antithrombin therapy. Blood Coagul fibrinolysis an Int J Haemost Thromb. 2014;25(5):444-50. http://dx.doi.org/0.1097/MBC.0000000000000076 10. Paidas MJ, Triche EW, James AH, DeSancho M, Robinson C, Lazarchick J, et al. Recombinant Human Antithrombin in Pregnant Patients with Hereditary Antithrombin Deficiency: Integrated Analysis of Clinical Data. Am J Perinatol. 2016;33(4):343-9. http://dx.doi.org/10.1055/s$\underline{0035-1564423}$

11. Rhéaume M, Weber F, Durand M, Mahone M. Pregnancy-Related Venous Thromboembolism Risk in Asymptomatic Women With Antithrombin Deficiency: A Systematic Review. Obstet Gynecol. 2016;127(4):649-56. http://dx.doi.org/10.1097/AOG.000000000000134 7

12. Ilonczai P, Oláh Z, Selmeczi A, Kerényi A, Bereczky Z, Póka R, et al. Management andoutcome of pregnancies in women with antithrombin deficiency: a single-center experience and review of literature. Blood Coagul fibrinolysis an Int $J$ Haemost Thromb. 2015;26(7):798-804.

http://dx.doi.org/10.1097/MBC.000000000000034 
Revista de Ensino, Ciência e Inovação em Saúde v.2, n.1 (2021) 63-68

13. Martinelli N, Girelli D, Baroni M, Guarini P, Sandri M, Lunghi B, et al. Activated factor VIIantithrombin complex predicts mortality in patients with stable coronary artery disease: a cohort study. J Thromb Haemost. 2016;14(4):655-66.

http://dx.doi.org/10.1111/jth.13274
14. Mendes-Da-Silva W. Contribuições e Limitações de Revisões Narrativas e Revisões Sistemáticas na Área de Negócios. Rev Adm Contemp. 2019;23(2):1-11. http://dx.doi.org/10.1590/1982$\underline{7849 \mathrm{rac} 2019190094}$ 